University of Wollongong

Research Online

Faculty of Engineering and Information

Faculty of Engineering and Information

Sciences - Papers: Part A

Sciences

$1-1-2015$

Symmetries and solutions of the non-autonomous von Bertalanffy equation

Maureen P. Edwards

University of Wollongong, maureen@uow.edu.au

R Anderssen

CSIRO Mathematics

Follow this and additional works at: https://ro.uow.edu.au/eispapers

Part of the Engineering Commons, and the Science and Technology Studies Commons

Research Online is the open access institutional repository for the University of Wollongong. For further information contact the UOW Library: research-pubs@uow.edu.au 


\title{
Symmetries and solutions of the non-autonomous von Bertalanffy equation
}

\begin{abstract}
For growth in a closed environment, which is indicative of the situation in laboratory experiments, autonomous ODE models do not necessarily capture the dynamics under investigation. The importance and impact of a closed environment arise when the question under examination relates, for example, to the number of the surviving microbes, such as in a study of the spoilage and contamination of food, the gene silencing activity of fungi or the production of a chemical compound by bacteria or fungi. Autonomous ODE models are inappropriate as they assume that only the current size of the population controls the growth-decay dynamics. This is reflected in the fact that, asymptotically, their solutions can only grow or decay monotonically or asymptote. Non-autonomous ODE models are not so constrained. A natural strategy for the choice of non-autonomous ODEs is to take appropriate autonomous ones and change them to be non-autonomous through the introduction of relevant non-autonomous terms. This is the approach in this paper with the focus being the von Bertalanffy equation. Since this equation has independent importance in relation to practical applications in growth modelling, it is natural to explore the deeper relationships between the introduced non-autonomous terms through a symmetry analysis, which is the purpose and goal of the current paper. Infinitesimals are derived which allow particular forms of the non-autonomous von Bertalanffy equation to be transformed into autonomous forms for which some new analytic solutions have been found.
\end{abstract}

\section{Keywords}

von, equation, autonomous, solutions, symmetries, bertalanffy, non

Disciplines

Engineering | Science and Technology Studies

\section{Publication Details}

Edwards, M. \& Anderssen, R. S. (2015). Symmetries and solutions of the non-autonomous von Bertalanffy equation. Communications in Nonlinear Science and Numerical Simulation, 22 (1-3), 1062-1067. 


\title{
Symmetries and solutions of the non-autonomous von Bertalanffy equation
}

\author{
Maureen P. Edwards ${ }^{\mathrm{a}, *}$, Robert S. Anderssen ${ }^{\mathrm{b}}$ \\ ${ }^{a}$ School of Mathematics and Applied Statistics, University of Wollongong, NSW 2522, Australia \\ ${ }^{b}$ CSIRO Mathematics, Informatics and Statistics, GPO Box 664, Canberra, ACT 2601, Australia
}

\begin{abstract}
For growth in a closed environment, which is indicative of the situation in laboratory experiments, autonomous ODE models do not necessarily capture the dynamics under investigation. The importance and impact of a closed environment arise when the question under examination relates, for example, to the number of the surviving microbes, such as in a study of the spoilage and contamination of food, the gene silencing activity of fungi or the production of a chemical compound by bacteria or fungi.

Autonomous ODE models are inappropriate as they assume that only the current size of the population controls the growth-decay dynamics. This is reflected in the fact that, asymptotically, their solutions can only grow or decay monotonically or asymptote. Non-autonomous ODE models are not so constrained. A natural strategy for the choice of non-autonomous ODEs is to take appropriate autonomous ones and change them to be non-autonomous through the introduction of relevant non-autonomous terms. This is the approach in this paper with the focus being the von Bertalanffy equation.

Since this equation has independent importance in relation to practical applications in growth modelling, it is natural to explore the deeper relationships between the introduced non-autonomous terms through a symmetry analysis, which is the purpose and goal of the current paper. Infinitesimals are derived which allow particular forms of the non-autonomous von Bertalanffy equation to be transformed into autonomous forms for which some new analytic solutions have been found.
\end{abstract}

Keywords: Autonomous, non-autonomous, ordinary differential equations, von Bertalanffy, Lie symmetries, closed-form solutions

\section{Introduction}

For the simulation of general growth-decay dynamics, the non-autonomous von Bertalanffy equation

$$
\frac{d N}{d t}=\alpha(t) N^{\beta}-a(t) N^{b}, \quad N=N(t)
$$

\footnotetext{
${ }^{*}$ Corresponding author. Tel.: +61242214768

Email addresses: maureen@uow. edu . au (Maureen P. Edwards), Bob. Anderssen@csiro.au (Robert S. Anderssen) 
with (without loss of generality) $\alpha(t), \beta, a(t)$ and $b$ non-negative, has been proposed by Edwards $e t$ $a l$. [1] as a model of the interaction of the current size $N(t)$ of a population with the environment in which it is living. In this paper, we investigate the symmetry properties of a generalisation of (1), which includes (1) as a special case, where a forcing term $\psi(t)$ is added to obtain

$$
\frac{d N}{d t}=\alpha(t) N^{\beta}-a(t) N^{b}+\psi(t), \quad N=N(t)
$$

Exact solutions to some specific forms of (1) and (2), for $\beta, b \in\{0,1,2,3\}$ and special forms for $\alpha(t), a(t)$ and $\psi(t)$ are well documented [2, 3, 4]. In particular, the solution of a linear form of (1) with $\beta=b=1$ has been proposed by Peleg and Corradini [5] as representative of a multiplicative population growth/decay process.

The paper has been organized in the following manner. Background about earlier published research is given in Section 2. The Lie point symmetries and associated infinitesimals for equation (2) are derived in Section 3. Utilization of the infinitesimals to derive new results is preformed in Section 4. Conclusions are given in Section 5.

\section{Background}

As explained by Edwards et al. [1] and others such as Coleman [6], the formulation of models for the growth of microbes (fungi; bacteria) must not only take account of the current number of the microbes but also of the effect of the environment in which the growth is occurring and of the type of measurements used to record the growth.

Consequently, autonomous ODE models are not always appropriate. For example, for the surviving members in a population, the growth will eventually attain a maximum and then decay. Thus, for the modelling of growth in a closed environment, non-autonomous ODEs become an appropriate choice with the role of the environment determining the structure of the nonautonomous terms.

The symmetry properties of some special forms of (2) have already been published. For example, when $\psi(t)=0$ and $b=1$, (2) becomes the Bernoulli equation which can be linearised and consequently solved [4]. A symmetry analysis for the Bernoulli equation is given in Ibragimov [7]. Taking $\beta=2$ and $b=1$ recovers the Riccati equation, for which a symmetry analysis can be found in Ibragimov [7] and has also been examined by Cheb-Terrab and Kolokolnikov [8]. When $\beta=3$ and $b \in\{1,2\}$, equation (2) corresponds to an Abel equation of the first kind. Its symmetry properties have been examined by Schwarz [9], as well as Ibragimov [7] and Cheb-Terrab and Kolokolnikov [8]. The case $b=1$ and $\beta \in \mathbb{N}$ corresponds to (2) being a polynomial in the dependent variable $N$. It is sometimes referred to as the Chini equation [8] since a symmetry analysis of this form of (2) was performed by Chini [10]. Schwarz [9] also considers the symmetries of the generalised Abel's equation

$$
y^{\prime}+\sum_{k=1}^{K} a_{k}(x) y^{k}=0
$$

for $K>3$ and $a_{K}(x) \neq 0$, which includes (2) for $\beta, b \in \mathbb{N}$.

In this paper, the only restriction placed on $\beta$ and $b$ are that they are non-negative. In particular, there is no constraint that the right hand side of (2) must be a polynomial in the dependent variable $N$. 


\section{Lie point symmetries}

The Lie symmetry group method [11] is used to obtain the Lie point symmetry generators of (2). There are many excellent texts devoted to this topic $[12,13,14,15]$. We seek the transformation

$$
t_{1}=t+\epsilon \tau(t, N)+O\left(\epsilon^{2}\right), \quad N_{1}=N+\epsilon \eta(t, N)+O\left(\epsilon^{2}\right)
$$

that leaves the governing equation (2) invariant. The symbol of the first prolongation of the group (3) is

$$
\Gamma^{(1)}=\tau \frac{\partial}{\partial t}+\eta \frac{\partial}{\partial N}+\eta_{[t]} \frac{\partial}{\partial N^{\prime}}
$$

where $\eta_{[t]}=D(\eta)-D(\tau) N^{\prime}$ and $D$ is the total derivative operator given by

$$
D=\frac{\partial}{\partial t}+N^{\prime} \frac{\partial}{\partial N}+N^{\prime \prime} \frac{\partial}{\partial N^{\prime}}+\cdots
$$

Taking the first prolongation (4) of (2) and using the governing equation to eliminate the derivative $\frac{d N}{d t}$ gives a single determining equation. The functions $\tau$ and $\eta$ are independent of the derivatives of $N$. However, because the determining equation is first order, it will not split into an overdetermined system of linear determining equations. Following Ibragimov [7], it is assumed that $\tau$ and $\eta$ are linear functions of $N$, that is,

$$
\tau(t, N)=F_{1}(t) N+F_{0}(t), \quad \eta(t, N)=G_{1}(t) N+G_{0}(t) .
$$

The determining equation becomes

$$
\begin{gathered}
\left(G_{1}(t) \alpha(t)-\frac{d F_{0}}{d t} \alpha(t)-\frac{d \alpha}{d t} F_{0}(t)-2 F_{1}(t) \alpha(t) \psi(t)-\alpha(t) \beta G_{1}(t)\right) N^{\beta} \\
+\left(2 F_{1}(t) a(t) \psi(t)-G_{1}(t) a(t)+\frac{d a}{d t} F_{0}(t)+\frac{d F_{0}}{d t} a(t)+a(t) b G_{1}(t)\right) N^{b} \\
\quad-\left(\frac{d \alpha}{d t} F_{1}(t)+\frac{d F_{1}}{d t} \alpha(t)\right) N^{\beta+1}+\left(\frac{d F_{1}}{d t} a(t)+\frac{d a}{d t} F_{1}(t)\right) N^{b+1} \\
+2 F_{1}(t) \alpha(t) a(t) N^{\beta+b}-F_{1}(t)(\alpha(t))^{2} N^{2 \beta}-F_{1}(t)(a(t))^{2} N^{2 b} \\
-\alpha(t) \beta G_{0}(t) N^{\beta-1}+a(t) b G_{0}(t) N^{b-1}+\left(\frac{d G_{1}}{d t}-\frac{d F_{1}}{d t} \psi(t)-\frac{d \psi}{d t} F_{1}(t)\right) N \\
+\frac{d G_{0}}{d t}+G_{1}(t) \psi(t)-\frac{d F_{0}}{d t} \psi(t)-F_{1}(t)(\psi(t))^{2}-\frac{d \psi}{d t} F_{0}(t)=0 .
\end{gathered}
$$

At this stage it is normally assumed that the powers of $N$ are independent which means that the coefficients of the powers of $N$ are equated to zero. This leads to a set of equations which gives relationships between the functions defining $\tau$ and $\eta$. In the current situation this immediately implies that $F_{1}(t)=G_{0}(t)=0$. It then follows that $G_{1}(t)=a_{1}, a_{1} \in \mathbb{R}$. The remaining equations left to be solved are

$$
\begin{gathered}
a_{1} \alpha(t)(1-\beta)-\frac{d F_{0}}{d t} \alpha(t)-\frac{d \alpha}{d t} F_{0}(t)=0, \\
a_{1} a(t)(1-b)-\frac{d F_{0}}{d t} a(t)-\frac{d a}{d t} F_{0}(t)=0, \\
a_{1} \psi(t)-\frac{d F_{0}}{d t} \psi(t)-\frac{d \psi}{d t} F_{0}(t)=0 .
\end{gathered}
$$


The advantage of this set of equations is that they can be exploited to derive solutions to (2) by taking advantage of the properties of $\alpha(t), a(t)$ and $\psi(t)$.

\subsection{The $\psi(t) \not \equiv 0$ case}

If it assumed that $\psi(t) \not \equiv 0$, then solving (6c) gives

$$
F_{0}(t)=\frac{a_{1} \int \psi(t) d t+c_{1}}{\psi(t)}, \quad c_{1} \in \mathbb{R} .
$$

It follows from (6a) and (6b) that

$$
\alpha(t)=\frac{c_{2} \psi(t)}{\left(a_{1} \int \psi(t) d t+c_{1}\right)^{\beta}}, \quad a(t)=\frac{c_{3} \psi(t)}{\left(a_{1} \int \psi(t) d t+c_{1}\right)^{b}}, \quad c_{2}, c_{3} \in \mathbb{R} .
$$

Consequently, under these constraints, (2) admits the symmetry infinitesimals

$$
\tau(t, N)=\frac{a_{1} \int \psi(t) d t+c_{1}}{\psi(t)}, \quad \eta(t, N)=a_{1} N .
$$

\subsubsection{Non-independent powers of $N$}

Breaking the assumption that the powers of $N$ are independent may lead to special forms of (2) with additional symmetries. A number of these special cases were analysed and lead only to the infinitesimals (8). However, the case $\beta=1$ and $b=1 / 2$ did not fall into this category. For this case, the structure of (5) becomes overly complex and the analysis is difficult. However, considering equivalence transformations [16] allows one of the functions of $t$ in (2) to be set to 1. Choosing $\alpha(t)=1$ allows the remaining determining equations to be solved to give

$$
\begin{gathered}
a(t)=c_{1} e^{-t}, \quad F_{1}(t)=c_{2} e^{-t}, \quad F_{0}(t)=\left(c_{2} e^{-t}+c_{3} e^{2 t}\right) \psi(t), \\
G_{1}(t)=2\left(c_{2} e^{-t}+c_{3} e^{2 t}\right) \psi^{\prime}(t)+2 c_{3} e^{2 t} \psi(t), \quad G_{0}(t)=0,
\end{gathered}
$$

where $c_{1}, c_{2}, c_{3} \in \mathbb{R}$ and $\psi(t)$ satisfies the equation

$$
2\left(c_{2} e^{-t}+c_{3} e^{2 t}\right) \psi^{\prime \prime}-\left(4 c_{2} e^{-t}-5 c_{3} e^{2 t}\right) \psi^{\prime}+2 c_{3} e^{2 t} \psi=c_{1}^{2} c_{2} e^{-3 t} .
$$

This linear equation for $\psi(t)$ can be solved yielding an explicit expression which is highly complex.

Note that setting $c_{1}=0$ gives $a(t)=0$ with the consequence that (2) becomes linear. The case $c_{2} \neq 0$ results in a linear expression for $\tau(t, N)$ in $N$. This leads to new infinitesimals, the consequences of which relative to what is presented here needs independent investigation.

\subsection{The $\psi(t) \equiv 0$ case}

The infinitesimals (8) and the conditions (7) on $\alpha(t)$ and $a(t)$ are valid only when $\psi(t) \not \equiv 0$. When $\psi(t) \equiv 0$, the earlier constraint $F_{1}(t)=G_{0}(t)=0$ and $G_{1}(t)=a_{1}, a_{1} \in \mathbb{R}$, remains valid. Now (6c) is automatically satisfied. Assuming that $\alpha(t) \neq 0$ and $\beta \neq 1$, the remaining determining equations $(6 a)$ and $(6 b)$ yield

$$
F_{0}(t)=\frac{a_{1}(1-\beta) \int \alpha(t) d t+c_{1}}{4^{\alpha(t)}}
$$


and

$$
a(t)=c_{2} \alpha(t)\left(a_{1}(1-\beta) \int \alpha(t) d t+c_{1}\right)^{(b-\beta) /(\beta-1)},
$$

where $c_{1}, c_{2} \in \mathbb{R}$. (Note that the case $\beta=1$ will recover the Bernoulli equation, and is not considered here.) In this instance, the infinitesimals are

$$
\tau(t, N)=\frac{a_{1}(1-\beta) \int \alpha(t) d t+c_{1}}{\alpha(t)}, \quad \eta(t, N)=a_{1} N .
$$

\subsection{The $\psi(t)=0$ for some $t \in \mathbb{R}($ but $\psi(t) \not \equiv)$ case}

If $\psi(t)=0$ anywhere, equation (6c) cannot be used. From (6), a solution can be found for $F_{0}(t)$ in terms of either $\alpha(t)$ or $a(t)$ which strongly restricts the form that $\psi(t)$ can have. As in the previous case 3.2, solving (6a) followed by (6b) gives (9) and (10) for $F_{0}(t)$ and $a(t)$, respectively. Consquently, (6c) gives

$$
\psi(t)=\frac{c_{3} \alpha(t)}{\left(a_{1}(1-\beta) \int \alpha(t)+c_{1}\right)^{\beta /(\beta-1)}}, \quad c_{3} \in \mathbb{R} .
$$

We can now only solve (5) for very special forms of $\psi(t)$ which are determined by the choice of either $\alpha(t)$ or $a(t)$. This establishes that (5) can not be solved for arbitrary $\psi(t)$ when there exists a $\hat{t}$ for which $\psi(\hat{t})=0$. Because the determining equation (5) can no longer be solved for arbitrary $\psi(t)$, this situation is not pursued further in this paper.

\section{Symmetry reductions and solutions of the non-autonomous von Bertalanffy equation}

The canonical coordinates corresponding to the symmetry infinitesimals (8) for $\psi(t) \neq 0$ are

$$
u=\frac{N}{a_{1} \int \psi(t) d t+c_{1}}, \quad v=\frac{1}{a_{1}} \ln \left(a_{1} \int \psi(t) d t+c_{1}\right), \quad a_{1} \neq 0,
$$

which reduce the non-autonomous von Bertalanffy equation (2) to the autonomous equation

$$
\frac{d u}{d v}=c_{2} u^{\beta}-c_{3} u^{b}-a_{1} u+1 .
$$

This transformed equation is valid irrespective of the values of $\beta, b, a_{1}, c_{1}$ and $c_{2}$ and is independent of the form of the forcing function $\psi(t)$. The only constraint is that $\alpha(t)$ and $a(t)$ are determined by $\psi(t)$ as detailed in (7). Any solution of (13) will lead to a solution for (2). Table 1 gives the form of $\alpha(t)$ and $a(t)$ and the corresponding canonical coordinates $u$ and $v$ for some simple functional forms of $\psi(t)$.

There is freedom in the choice of $c_{2}, c_{3}$ and $a_{1}$ and the powers $\beta$ and $b$ in solving (13). For example, taking $\beta=b+1$ and $c_{2}=c_{3} a_{1}$ means that right hand side of (13) can be factorized and so (13) can be written as

$$
v=\int \frac{d u}{\left(c_{3} u^{b}-1\right)\left(a_{1} u-1\right)}+C,
$$

with $C \in \mathbb{R}$ the constant of integration. The cases $b=1$ and 2 (with corresponding values $\beta=2$ and 3) are not of direct interest as they correspond to the Riccati or Abel equations. For some values of $b$, equation (14) can be solved analytically. 


\begin{tabular}{|c|c|c|c|c|}
\hline$\psi(t)$ & $\alpha(t)$ & $a(t)$ & $u$ & $v$ \\
\hline$c_{4}$ & $\frac{c_{2} c_{4}}{\left(a_{1} c_{4} t+c_{1}\right)^{\beta}}$ & $\frac{c_{3} c_{4}}{\left(a_{1} c_{4} t+c_{1}\right)^{b}}$ & $\frac{N}{a_{1} c_{4} t+c_{1}}$ & $\frac{1}{a_{1}} \ln \left(a_{1} c_{4} t+c_{1}\right)$ \\
\hline$c_{4} t^{m}, m \neq-1$ & $\frac{c_{2} c_{4} t^{m}}{\left(\frac{a_{1} c_{4}}{m+1} t^{m+1}+c_{1}\right)^{\beta}}$ & $\frac{c_{3} c_{4} t^{m}}{\left(\frac{a_{1} c_{4}}{m+1} t^{m+1}+c_{1}\right)^{b}}$ & $\frac{N}{\frac{a_{1} c_{4}}{m+1} t^{m+1}+c_{1}}$ & $\frac{\ln \left(\frac{a_{1} c_{4}}{m+1} t^{m+1}+c_{1}\right)}{a_{1}}$ \\
\hline$c_{4} t^{-1}$ & $\frac{c_{2} c_{4}}{t\left(a_{1} c_{4} \ln t+c_{1}\right)^{\beta}}$ & $\frac{c_{3} c_{4}}{t\left(a_{1} c_{4} \ln t+c_{1}\right)^{b}}$ & $\frac{N}{a_{1} c_{4} \ln t+c_{1}}$ & $\frac{\ln \left(a_{1} c_{4} \ln t+c_{1}\right)}{a_{1}}$ \\
\hline$c_{4} e^{m t}, m \neq 0$ & $\frac{c_{2} c_{4} e^{m t}}{\left(\frac{a_{1} c_{4}}{m} e^{m t}+c_{1}\right)^{\beta}}$ & $\frac{c_{3} c_{4} e^{m t}}{\left(\frac{a_{1} c_{4}}{m} e^{m t}+c_{1}\right)^{b}}$ & $\frac{N}{\frac{a_{1} c_{4}}{m} e^{m}+c_{1}}$ & $\frac{\ln \left(\frac{a_{1} c_{4}}{m} e^{m}+c_{1}\right)}{a_{1}}$ \\
\hline$c_{4}\left(1-e^{m t}\right), m \neq 0$ & $\frac{c_{2} c_{4}\left(1-e^{m t}\right)}{\left(a_{1} c_{4}\left(t-\frac{1}{m} e^{m t}\right)+c_{1}\right)^{\beta}}$ & $\frac{c_{3} c_{4}\left(1-e^{m t}\right)}{\left(a_{1} c_{4}\left(t-\frac{1}{m} e^{m t}\right)+c_{1}\right)^{b}}$ & $\frac{N}{a_{1} c_{4}-\frac{1}{m} e^{m}+c_{1}}$ & $\frac{\ln \left(a_{1} c_{4}-\frac{1}{m} e^{m}+c_{1}\right)}{a_{1}}$ \\
\hline
\end{tabular}

Table 1: Functional forms of $\psi(t)$ with corresponding forms of $\alpha(t), a(t)$ and canonical coordinates $u$ and $v$.

\subsection{Case $b=3(\beta=4)$}

For $b=3(\beta=4)$, the closed form solution of (14) is given by

$$
\begin{aligned}
v= & \frac{c_{3}^{2 / 3}}{6\left(a_{1}^{3}-c_{3}\right)}\left[-\frac{6 a_{1}^{2}}{c_{3}^{2 / 3}} \ln \left(a_{1} u-1\right)+2\left(1+\frac{a_{1}}{c_{3}^{1 / 3}}\right) \ln \left(u-\frac{1}{c_{3}^{1 / 3}}\right)+\frac{2 a_{1}^{2}}{c_{3}^{2 / 3}} \ln \left(c_{3} u^{3}-1\right)\right. \\
& \left.-\left(1+\frac{a_{1}}{c_{3}^{1 / 3}}\right) \ln \left(u^{2}+\frac{u}{c_{3}^{1 / 3}}+\frac{1}{c_{3}^{2 / 3}}\right)+2 \sqrt{3}\left(-1+\frac{a_{1}}{c_{3}^{1 / 3}}\right) \tan ^{-1}\left(\frac{c_{3}^{1 / 3}}{\sqrt{3}}\left(2 u+\frac{1}{c_{3}^{1 / 3}}\right)\right)\right]+C,
\end{aligned}
$$

where $c_{3} \neq a_{1}^{3}$. In the case that $c_{3}=a_{1}^{3}$, the closed form solution is given by

$$
v=\frac{1}{3 a_{1}}\left[-\ln \left(a_{1} u-1\right)-\frac{1}{a_{1} u-1}+\frac{1}{2} \ln \left(a_{1}^{2} u^{2}+a_{1} u+1\right)+\frac{1}{\sqrt{3}} \tan ^{-1}\left(\frac{1}{\sqrt{3}}\left(2 a_{1} u+1\right)\right)\right]+C .
$$

\subsection{Case $b=4(\beta=5)$}

When $b=4(\beta=5)$, the closed form solution of (14) is given by

$$
\begin{aligned}
v= & -\frac{c_{3}^{1 / 4}}{4\left(a_{1}^{4}-c_{3}\right)}\left[\frac{4 a_{1}^{3}}{c_{3}^{1 / 4}} \ln \left(a_{1} u-1\right)+\left(\sqrt{c_{3}}+a_{1}^{2}\right) \ln \left(\frac{u+\frac{1}{c_{3}^{1 / 4}}}{u-\frac{1}{c_{3}^{1 / 4}}}\right)\right. \\
& \left.+2\left(\sqrt{c_{3}}-a_{1}^{2}\right) \tan ^{-1}\left(c_{3}^{1 / 4} u\right)-\frac{a_{1}^{3}}{c_{3}^{1 / 4}} \ln \left(c_{3} u^{4}-1\right)-a_{1} c_{3}^{1 / 4} \ln \left(\frac{1-\sqrt{c_{3}} u^{2}}{1+\sqrt{c_{3}} u^{2}}\right)\right]+C,
\end{aligned}
$$

where $c_{3} \neq a_{1}^{4}$. When $c_{3}=a_{1}^{4}$, the solution takes the form

$$
v=\frac{1}{4 a_{1}}\left[\frac{3}{2} \ln \left(a_{1} u-1\right)-\frac{1}{a_{1} u-1}+\frac{1}{2} \ln \left(a_{1}^{2} u^{2}+1\right)+\tan ^{-1}\left(a_{1} u\right)+\frac{1}{2} \ln \left(a_{1} u+1\right)\right]+C .
$$


4.3. Case $b=1 / 2(\beta=3 / 2)$

With $b=1 / 2(\beta=3 / 2)$, the closed form solution of (14) is

$$
v=\frac{-\sqrt{a_{1}} \ln \left(a_{1} u-1\right)+2 \sqrt{a_{1}} \ln \left(c_{3} \sqrt{u}-1\right)+2 c_{3} \tanh ^{-1}\left(\sqrt{a_{1} u}\right)}{\sqrt{a_{1}}\left(a_{1}-c_{3}^{2}\right)}+C,
$$

where $a_{1} \neq c_{3}^{2}$. When $a_{1}=c_{3}^{2}$, the solution takes the form

$$
v=\frac{1}{c_{3}^{2}}\left[\frac{1}{c_{2} \sqrt{u}-1}+\frac{1}{2} \ln \left(c_{3} \sqrt{u}-1\right)-\frac{1}{2}\left(c_{3} \sqrt{u}+1\right)\right]+C .
$$

In terms of the original variables, the solutions (19) and (20) are

$$
\begin{aligned}
\frac{\left(a_{1}-c_{3}^{2}\right)}{\sqrt{a_{1}}} \ln \left(a_{1} \int \psi(t) d t+c_{1}\right)= & -\sqrt{a_{1}} \ln \left(\frac{a_{1} N}{a_{1} \int \psi(t) d t+c_{1}}-1\right)+2 \sqrt{a_{1}} \ln \left(c_{3} \sqrt{\frac{N}{a_{1} \int \psi(t) d t+c_{1}}}-1\right) \\
& +2 c_{3} \tanh ^{-1}\left(\sqrt{\frac{a_{1} N}{a_{1} \int \psi(t) d t+c_{1}}}\right)+C, \quad a_{1} \neq c_{3}^{2},
\end{aligned}
$$

and

$$
\begin{aligned}
\ln \left(c_{3}^{2} \int \psi(t) d t+c_{1}\right)= & \frac{1}{c_{2} \sqrt{\frac{N}{c_{3}^{2} \int \psi(t) d t+c_{1}}}-1}+\frac{1}{2} \ln \left(c_{3} \sqrt{\frac{N}{c_{3}^{2} \int \psi(t) d t+c_{1}}}-1\right) \\
& -\frac{1}{2}\left(c_{3} \sqrt{\frac{N}{c_{3}^{2} \int \psi(t) d t+c_{1}}}+1\right)+C, \quad a_{1}=c_{3}^{2} .
\end{aligned}
$$

Solutions (21) and (22) are valid for any form of $\psi(t)$ and satisfy the von Bertalanffy equation

$$
\frac{d N}{d t}=\frac{c_{2} \psi(t)}{\left(a_{1} \int \psi(t) d t+c_{1}\right)^{3 / 2}} N^{3 / 2}-\frac{c_{3} \psi(t)}{\left(a_{1} \int \psi(t) d t+c_{1}\right)^{1 / 2}} N^{1 / 2}+\psi(t) .
$$

Importantly, this pair of solutions is new. They are independent of the earlier published results from symmetry analyses discussed in section 2 because, for $b=1 / 2(\beta=3 / 2)$, equation (2) is no longer a polynomial in $N$.

Other closed form solutions for the canonical coordinate $v$ in terms of $u$ are possible depending on the chosen values of the powers $\beta, b$ and the constants $a_{1}, c_{2}$ and $c_{3}$. Once a specific solution for the canonical coordinates has been found and a functional form for $\psi(t)$ has been chosen, the corresponding forms of the infinitesimals $\tau(t, N)$ and $\eta(t, N)$ in Table 1 can be used. This will yield the closed form solution of (2) for the appropriate growth and decay functions $\alpha(t)$ and $a(t)$ generated by the choice of $\psi(t)$.

In the case $\psi(t) \equiv 0$, the canonical coordinates corresponding to the symmetry infinitesimals (11) are

$u=\frac{N}{\left(a_{1}(1-\beta) \int \alpha(t) d t+c_{1}\right)^{1 /(1-\beta)}}, \quad v=\frac{1}{a_{1}(1-\beta)} \ln \left(a_{1}(1-\beta) \int \alpha(t) d t+c_{1}\right), \quad a_{1} \neq 0, \beta \neq 1$, 
which reduces the von Bertalanffy equation $(2)($ with $\psi(t)=0)$ to the autonomous equation

$$
\frac{d u}{d v}=u^{\beta}-c_{2} u^{b}-a_{1} u
$$

Note that for the case $\beta=1$, (2) corresponds to the Bernoulli equation which is linearisable and so is not considered in this paper.

\section{Conclusions}

In this work, we have used Lie symmetry methods to determine the constraints on the functions $\alpha(t), a(t)$ and $\psi(t)$ which allow nontrivial symmetries to exist for the non-autonomous von Bertalanffy equation (2). We have exploited these symmetry properties to construct new closed form solutions. This has extended the work of earlier authors who have previously considered various forms of the generalised Abel equation which correspond to (2) only when the right hand side is polynomial in the dependent variable $N$.

\section{Acknowledgements}

The authors would like to thank Ulrike Schumann, CSIRO Plant Industry, for introducing them to the background growth-decay problem for fungi which has led to this research. The authors would also like to thank the reviewer for the insightful observations which have led to a more complete analysis of the von Bertalanffy equation.

\section{References}

[1] Edwards, M., Schumann, U., Anderssen, R.. Modelling microbial growth in a closed environment. Journal of Math-for-Industry 2013;5:33-40.

[2] Murphy, G.. Ordinary Differential Equations and their Solutions. New York, Van Nostrand; 1960.

[3] Kamke, E.. Differentialgleichungen : Lösungsmethoden und Lösungen. Chelsea, New York; 1971.

[4] Polyanin, A., Zaitsev, V.. Handbook of Exact Solutions for Ordinary Differential Equations. CRC Press, Boca Raton; 1995.

[5] Peleg, M., Corradini, M.G.. Microbial Growth Curves: What the Models Tell Us and What They Cannot. Critical Reviews in Food Science and Nutrition 2011;51(10):917-945. doi:10.1080/10408398.2011.570463.

[6] Coleman, B.. Nonautonomous logistic equations as models of the adjustment of populations to environmental change. Mathematical Biosciences 1979;45:159-173.

[7] Ibragimov, N.. CRC Handbook of Lie Group Analysis of Differential Equations, Volume I: Symmetries, Exact Solutions, and Conservation Laws. CRC Press, Boca Raton; 1994.

[8] Cheb-Terrab, E., Kolokolnikov, T.. First-order ordinary differential equations, symmetries and linear transformations. European Journal of Applied Mathematics 2003;14:231-246.

[9] Schwarz, F.. Symmetry Analysis of Abel's Equation. Studies in Applied Mathematics 1998;100:269-294.

[10] Chini, M.. Sullintegrazione di alcune equazioni differenziali del primo ordine. Rendiconti Instituto Lombardo 1924;57(2):506-511.

[11] Lie, S.. Über die Integration durch bestimmte Integrale von einer Klasse linearer partieller Differentialgleichungen. Arch for Math 1881;6:328-368.

[12] Bluman, G., Cole, J.. Symmetry Methods for Differential Equations. Spring-Verlag, Berlin; 1974.

[13] Bluman, G., Kumei, S.. Symmetries and differential equations. Spring-Verlag, Berlin; 1989.

[14] Hill, J.. Differential Equations and Group Methods for Scientists and Engineers. CRC Press, Boca Raton; 1992.

[15] Olver, P.. Applications of Lie Groups to Differential Equations. Spring-Verlag, Berlin; 1986.

[16] Ovsiannikov, L.. Group Analysis of Differential Equations. Academic Press, New York; 1982. 